\title{
Introducción al dossier: Los organismos de inteligencia en Argentina. Miradas desde los archivos a una burocracia secreta
}

\author{
María Eva Muzzopappa \\ emuzzopappa@unrn.edu.ar \\ Instituto de Investigaciones en Diversidad y Procesos \\ de Cambio (IIDyPCa), Universidad Nacional de Río \\ Negro (UNRN) / CONICET, Argentina

\section{Mariana Nazar} \\ mariananazar@gmail.com \\ Dirección de Gestión del Conocimiento, \\ Investigaciones y Publicaciones del Instituto Nacional \\ de la Administración Pública (INAP), Escuela de \\ Política y Gobierno, Universidad Nacional de San \\ Martín (EpyG-UNSAM) / Asociación de Archivistas \\ en la Función Pública Argentina (AFPA), Argentina
}

En abril de 2021, la Comisión Bicameral de Fiscalización de los Organismos de Inteligencia del Congreso Nacional en Argentina -creada hace veinte años con la finalidad de fiscalizar a los organismos pertenecientes al Sistema de Inteligencia Nacional- presentó un informe que suscitó un escándalo mediático en materia de actividades ilegales de espionaje. La información daba cuenta de la forma en que, a lo largo de al menos cuatro años, se había montado una "estructura estatal paralela y clandestina: una verdadera organización mafiosa" integrada por la Agencia Federal de Informaciones (AFI) y el Servicio Penitenciario Federal, entre otros organismos estatales, y había contado con la participación de algunos medios de comunicación hegemónicos como difusores de esta información (Bertoia, 2021). 
Como si se tratara de un signo de época, también en otros países de la región se han presentado situaciones que evidencian el oscuro y arbitrario accionar de las agencias de inteligencia. Su aparición en clave de lo que Oszlak y O’Donnell definieron como una “cuestión socialmente problematizada” (1976) nos permite estimar que no se trata sólo de un interés por los ribetes escandalosos de cada uno de los casos, sino de la instalación de un debate respecto de sus lineamientos, objetivos, y mecanismos de control institucional.

En Chile, el cuestionamiento ha estado dirigido a la Dirección de Inteligencia de Carabineros (DIPOLCAR). En 2018, se develó que en el marco de la denominada "Operación Huracán" -una investigación sobre supuestas acciones terroristas de integrantes de comunidades mapuche- las pruebas aportadas a la justicia por esta Dirección consistían en una serie de montajes con el fin de inculpar a personas específicas. Un año después, y en el marco del llamado levantamiento o estallido social, un hackeo informático dejó disponible y en línea documentación que mostraba que Carabineros realizaba seguimientos a dirigentes estudiantiles, sindicales, ambientales, y que redactaba informes sobre actos, movilizaciones y conmemoraciones de diversa índole (Fossa, 2019; Sepúlveda, 2019). En esta oportunidad, las categorías utilizadas para identificar personas y grupos como objetivos de seguimiento y sospecha y las prácticas de malversación a la hora de realizar informes para presentar ante la Justicia fueron los objetos de un fuerte repudio social, que no puede dejar de desvincularse con las prácticas violentas del Cuerpo de Carabineros frente a la protesta social. El cuestionamiento, en definitiva, ponía en el centro del debate la definición de los objetivos de la inteligencia policial, aun fuertemente guiados por una concepción de "policía política".

Finalmente, podemos citar a Colombia, cuyo caso más emblemático fue el de las llamadas chuzadas (intercepción de comunicaciones, seguimientos y amenazas) realizadas por el Departamento Administrativo de Seguridad (DAS) durante el Gobierno de Álvaro Uribe en el año 2009. Un medio periodístico develó esta situación, la denuncia fue procesada por la justicia y finalmente la agencia fue disuelta dos años después. En enero de 2020, un nuevo caso de resonancia tuvo como protagonistas a varios oficiales de alto rango del Ejército, quienes fueron dados de baja tras comprobarse la realización de escuchas ilegales a políticos, magistrados, periodistas y a otros integrantes de las fuerzas armadas, para obtener "información de carácter personal".

La aparición en la escena pública de estos escándalos y el tratamiento que éstos suelen recibir, ya sea por parte de los medios de comunicación o los funcionarios y políticos, evidencia el profundo desconocimiento que existe sobre un sector del aparato estatal que se ha constituido y ha funcionado históricamente a partir del al to grado de autonomía que le otorga la puesta en acción de la categoría del secreto en general, y del secreto de estado en particular (Muzzopappa, 2018).

El secreto ha sido uno de los elementos centrales para el avance de técnicas y dispositivos de control y vigilancia. Estos organismos son sindicados como parte constitutiva de los estados modernos, en particular de sus procesos de racionalización de la política (L’Heuillet 2010). Al mismo tiempo, su relación primigenia con el secreto los sitúa en un espacio jurídico especial -hoy fuertemente puesto en tela de juicio- que ha inhibido estudios de tipo académico sobre su forma de organización, funcionamiento y prácticas, acotando fuertemente los análisis a los aspectos normativos de su desarrollo.

Sin embargo, hace alrededor de dos décadas que asistimos a un fenómeno que, aunque poco frecuente, ha permitido ampliar estas indagaciones. Se trata de la identificación y puesta a la consulta pública de un conjunto de archivos que comparten la característica de contener documentación que da cuenta de estas "actividades de inteligencia".

En Brasil, algunos de los archivos de Delegaciones/Departamentos estaduales de Orden Político y Social (DOPS) de la Policía Federal se encuentran resguardados en Archivos Públicos, mientras que otros se dice que fueron incinerados. El caso paradigmático es el de la DEOPS (1924-1983) de São Paulo, cuyo archivo fue transferido a la Policía Federal. Ante las limitaciones de acceso que dicha institución brindaba, y los reclamos sobre documentos que dieran cuenta de las desapariciones y muertes durante la Dictadura Militar, se generó un amplio debate social a partir del cual se terminó transfiriendo la documentación al Arquivo 
Público do Estado de São Paulo en el año 1991. En un principio, el acceso a los documentos fue restringido a familiares de presos y desaparecidos/as, en 1994 fue abierto a la consulta pública mediante la firma de un compromiso de responsabilidad. Entre 1991 y 1996, documentación de las delegaciones de Paraná, Ceará, Porto Alegre, Río de Janeiro, Guanabara, Maranhão y Espíritu Santos ha sido transferida a Archivos públicos estaduales. ${ }^{1}$ Asimismo, en el Arquivo Nacional se encuentra la documentación del Servicio Nacional de Informaciones (SIN, 1964-1974), del Consejo de Seguridad Nacional (CSN, 1964-1980), de la Comisión General de Investigaciones (CGI, 1968-1979), División de Inteligencia de Departamento (1964-1985), y de Divisiones de Seguridad e Informaciones de diversos organismos públicos (1964-1985).

En Paraguay, en el año 1992 fueron identificados los archivos de la policía secreta del dictador Stroessner en el marco de un allanamiento judicial. Los documentos, conocidos como los archivos del terror fueron trasladados al Palacio de Justicia, donde se encuentran bajo custodia del Museo de la Justicia-Centro de Documentación y Archivo para la Defensa de los Derechos Humanos

En Guatemala, la identificación de la extinta Policía Nacional (1960-1995) en un edificio casi abandonado, compuesta por 7900 metros lineales de documentos apilados en múltiples recovecos dió inició a un gran Proyecto de Recuperación que, contando con apoyo de la cooperación internacional, permitió la realización de un trabajo modélico en el aporte a la lucha contra la impunidad y la reparación de daños a las víctimas del genocidio en ese país. En Uruguay, se encuentra identificada y transferida a la Secretaría de Derechos Humanos de la Presidencia de la República la documentación de la Dirección Nacional de Información e Inteligencia (DNII) y de la Dirección General de Información e Inteligencia (DGII). En el Archivo General de la Nación de México, se encuentra abierta a la consulta pública la documentación de la Dirección Federal de Seguridad (DFS) y de la Dirección General de Investigaciones Políticas y Sociales (DGIPS), mientras se avanza en los procesos archivísticos para facilitar la consulta de la documentación del Centro de Investigación y Seguridad Nacional (CISEN). En el caso de Colombia, la documentación del ya mencionado Departamento Administrativo de Seguridad (DAS) se encuentra bajo custodia del Archivo General de la Nación, aunque fuera de consulta Pública. Por último, en el caso de Chile fue identificada documentación de inteligencia paraestatal producida en Colonia Dignidad. El fondo Sociedad Benefactora y Educacional Colonia Dignidad (1960-2005) fue descubierto en allanamientos policiales quedando bajo custodia de la Jefatura de Inteligencia Policial (JIPOL) hasta diciembre de 2017, cuando fueron requeridos por el Archivo Nacional para asegurar su preservación, encontrándose actualmente abiertos a la consulta pública. ${ }^{2}$

En el caso de Argentina, los archivos que se han identificado han sido producidos por las policías y los sistemas de inteligencia provinciales, las fuerzas armadas y fuerzas de seguridad nacionales. En su mayor parte, y con algunas excepciones, se trata de archivos fragmentados y sus condiciones de acceso son diversas: algunos se encuentran incautados como prueba judicial, otros han sido abiertos a la consulta pública, otros se encuentran en proceso de evaluación y varios de ellos transitan en un estado liminal donde la creación de instituciones o dependencias no se asocia a espacios físicos, personal ni tratamientos archivístico adecuado.

Esta situación ha permitido poner en escena dos grandes temas que, aunque se encuentran estrechamente relacionados, no siempre han sido trabajados de manera conjunta. El primero de ellos es el del tratamiento, acceso y gestión de estos archivos, que han pasado a tener una visibilidad inusitada por la importancia de su rol en el marco de las causas judiciales por crímenes de lesa humanidad. Así, pasaron a erigirse en una pieza fundamental en el proceso de Memoria, Verdad y Justicia de muy diversas maneras. En algunos casos, aportando datos referentes a personas detenidas-desaparecidas, y de manera creciente entregando información sobre el sistema represivo utilizado durante la última dictadura militar. Este tipo de aproximación al archivo ha permitido problematizar la recurrente mirada sobre el archivo como un depósito de datos que se encuentran allí sólo para ser hallados y certificados.

El segundo eje de gran relevancia en este campo es específicamente el análisis de los organismos estatales que han producido estos archivos: su marco normativo, organización, relaciones, prácticas, objetivos, categorías de uso y clasificación desde fines del siglo XIX hasta entrado el siglo XXI. A su vez, a la normativa se 
le deben añadir otras particularidades derivadas de la existencia -legal o regida por la costumbre- de una comunidad de inteligencia, de su actuación conjunta y en red, de las confrontaciones derivadas por los conflictos jurisdiccionales, así como de los períodos y temporalidades útiles para su estudio y análisis. Estas prácticas han legado un caudal de huellas que conllevan la necesidad de explicitar los recaudos metodológicos fundamentales a la hora de explotarlas como fuente de la investigación.

En ese sentido, los artículos reunidos en este dossier presentan la particularidad de aportar a este campo de estudios una perspectiva que recupera específicamente la pregunta por el funcionamiento, las relaciones y lógicas de trabajo de los organismos de inteligencia. Apuntando, a partir de la definición de casos y períodos temporales específicos, a pensar la constitución de estos organismos en una larga duración y como parte de la configuración de las burocracias estatales en Argentina.

Son resultado del proceso de trabajo conjunto del Proyecto de Investigación Científica y Tecnológica "Redes de Información. Archivos, lógicas y prácticas de los organismos de inteligencia en Argentina" (PICT 2018-0001917) financiado por la Agencia Nacional de Promoción de la Investigación, el Desarrollo Tecnológico y la Innovación, que reúne a un equipo de investigadoras/es abocadas/os al trabajo con un conjunto de fondos producidos por instituciones cuya función incluía la recopilación, búsqueda y análisis de información. Es esta una reflexión novedosa, ya que reúne y trasciende una diversidad de investigaciones que han tomado a estos archivos como eje de análisis. Esto pudo verse reflejado en la dificultad que tuvieron algunas de las evaluaciones para caracterizar el proyecto en su primera presentación. Mientras algunos de los comentarios apuntaban a considerar excesivamente "diverso" el corpus presentado, otros entendían que no estaban "suficientemente representadas" las distintas regiones del país. Si la segunda cuestión no era en modo alguno solucionable ya que se proponía el trabajo con los archivos disponibles, la primera hacía precisamente al núcleo de esta investigación. Así, a lo largo de las reuniones, y enfrentando el desafío de esa diversidad de materiales, instituciones y periodizaciones involucradas, nos dimos a la tarea de desarrollar los ejes considerados en este proyecto. En primer lugar, el dirigido a ampliar el conocimiento sobre el funcionamiento de los organismos de información e inteligencia desde una perspectiva que permitiera dar cuenta de su historicidad y de su relación con la política. En este proceso, fue necesario identificar las diferentes instituciones involucradas a partir de sus archivos, las misiones y funciones en el marco de las cuales se produjeron los documentos, mientras prestamos particular interés a la contextualización de las doctrinas sobre las cuales se montaron las categorías de orden, enemigo o seguridad, así como a las prácticas involucradas en la producción de la inteligencia y a las redes que fueron estableciéndose entre los distintos organismos y que dieron lugar a la comunidad informativa.

A fin de poder plantear la posibilidad de las comparaciones a lo largo del tiempo, las categorías utilizadas en los archivos fueron el primer eje sobre el cual se acordó un trabajo transversal, recuperando la especificidad de su contexto de enunciación y el período abordado. En este punto, consideramos importante rescatarlas en tanto categorías nativas, es decir, recuperar la literalidad de las expresiones que, en determinados momentos históricos y en agencias determinadas, se utilizaban para señalar o calificar a quienes eran objetivo de sus prácticas; para ello apuntamos a su reproducción textual, tal y como aparecían en el material de archivo. A partir de allí, podían ser distinguidas de aquellas otras introducidas por el análisis, cuyas categorías presentan gran cantidad de coincidencias, como en el caso de orden, seguridad y sospecha, entre otras que forman parte del arsenal conceptual de este campo de estudios.

En esta misma dirección, y apuntando a la contextualización histórica de los corpus, cada uno de los trabajos consideró el marco doctrinario general vigente al momento de creación de los archivos y documentos analizados, a fin de establecer los necesarios puntos de conexión y divergencia, pero también los anclajes para pensar las continuidades y quiebres.

Finalmente, los trabajos se centraron en dilucidar, a partir de las huellas que fueron apareciendo en los registros, las prácticas involucradas en la realización de tareas de inteligencia: recopilación de información de medios abiertos, seguimientos, relaciones y circuitos con otros organismos. 
Tal como se ha mencionado, el interés de los trabajos aquí reunidos se centra en los archivos. No en los documentos, meramente, sino en su organicidad y lógica subyacente, lo cual implica considerar desde la constitución de los organismos productores de tales archivos, la normativa que establece sus misiones y funciones, las rutinas y las prácticas de sus funcionarios, las categorías utilizadas para el ordenamiento y la caracterización de los sujetos que se encuentran bajo sospecha. Para citar a Ann Stoler (2010), el eje de la reflexión está puesto aquí en los archivos no como lugares de recuperación del conocimiento, sino de producción del mismo, y en la exploración de sus coordenadas de inteligibilidad. Porque, en definitiva, no se trata de establecer si el archivo "debe tratarse como un conjunto de normas discursivas, un proyecto utópico, un depósito de documentos, un corpus de enunciados o todas las anteriores" (Stoler, 2010, p. 476) sino considerarlos como aquellos lugares donde se oculta, revela y reproduce el poder estatal.

Es entonces que, en función de dicha propuesta, el dossier se inicia con el artículo de Mariana Nazar y Cecilia García Novarini quienes recuperan la centralidad de la perspectiva archivística para considerar la posibilidad de tal empresa. Para ello, primero presentan un detalle actualizado de los archivos de inteligencia actualmente disponibles en Argentina en función de su estado de conservación, tratamiento y acceso, así como los problemas asociados a la ausencia de una política pública archivística. Luego hacen una presentación de los principales conceptos de la disciplina subrayando la riqueza del paso de la información literal a la información contextual. Finalmente, presentan dos casos -en los cuales las autoras tuvieron activa participación- la Comisión Provincial por la Memoria y el del Archivo Nacional de la Memoria, que permiten ejemplificar el impacto de las decisiones de gestión de los archivos en las posibilidades del acceso a la información de los mismos.

Los siguientes artículos se presentan en una sucesión cronológica y cada uno de ellos se detiene en un período específico, a saber: 1930-1940; 1956-1976; 1969-1973 y 1983-2006.

El primero de ellos es el de Melisa Fernández Marrón y Pedro Berardi, un revelador aporte respecto a la génesis de los archivos de inteligencia y su estrecha relación con los archivos policiales a partir de la indagación sobre los mecanismos de investigación e información policial. En el trabajo dan cuenta de cómo se fueron definiendo formas de observación e intervención que irán configurando saberes y experiencias que, para mediados de la década de 1930, formarán parte del núcleo central de las prácticas de inteligencia. A partir del análisis comparativo entre la reorganización de la Sección de Orden Social de la Policía de la Provincia de Buenos Aires y su creación en la policía del entonces Territorio Nacional de Río Negro, presentan en detalle el contexto de conflictividad registrado por estas instituciones. A partir de allí, reconstruyen la asignación de funciones de cada una para pasar a detallar los procedimientos de recopilación de información, la confección de diferentes tipos de registros y la coordinación de actividades entre organismos, prácticas que van a ir definiendo un campo específico entre las burocracias estatales. De particular interés resulta la elaboración de los prontuarios de personas, que será la herramienta básica para el conocimiento del enemigo y cuyo formato trascenderá largamente este período. Finalmente, a partir del análisis de dos casos, abordan la utilización de aquellas categorías a partir de las cuales se asignan los sentidos de lo peligroso.

El siguiente artículo, de Julieta Sahade, Ingrid Jaschek y Magdalena Lanteri, aborda un archivo emblemático para el estudio de los organismos de inteligencia en el marco de las causas de lesa humanidad: el archivo de la Dirección de Inteligencia de la Policía de la Provincia de Buenos Aires. A partir del reconocimiento de su trayectoria, las autoras muestran la diversidad de sentidos que el mismo ha ido adquiriendo a lo largo de estos años, pudiendo trascender y complejizar su inicial caracterización de "archivo de la represión", así como presentar las posibilidades surgidas del avance del trabajo de puesta al acceso con perspectiva archivística. De tal manera que, recuperando la instancia fundacional de la institución en 1956, van estableciendo algunos puntos de relevancia en su desarrollo histórico, para detenerse en la reflexión sobre temas que han ido marcando una agenda de investigación respecto de las actividades de inteligencia: la constitución y conformación del archivo respecto de su "documentación heredada", la extensión de redes de alcance territorial más allá de los límites de la Provincia de Buenos Aires, su lugar en la articulación de 
la comunidad informativa, el detalle del ciclo de inteligencia y las numerosas prácticas desplegadas para la obtención de información.

También la pregunta por el circuito de producción de la información de inteligencia es retomada en el artículo de Mónica Gatica y Axel Binder, específicamente por la división del trabajo entre las distintas fuerzas y sus respectivos organismos. A partir de la idea de información como mercancía, la propuesta avanza en el análisis de un conjunto documental del período 1969-1973 del Servicio de Informaciones del Chubut (SICh), cuya característica principal reside en la profusa comunicación establecida entre diferentes organismos de inteligencia para la confección de los informes contenidos en la carpeta "Legajo $\mathrm{n}^{\circ} 15$ de Actividades Culturales". Los cuatro casos allí contenidos les permite avanzar sobre las categorías utilizadas y la densidad de la información obtenida, en diferentes momentos, por la Policía provincial, la Policía Federal, la Base Aeronaval Almirante Zar y la Secretaría de Informaciones del Estado (SIDE), la propia SICh y otras dependencias -como la Subsecretaría de Educación y Justicia- para la producción de inteligencia. En este sentido, el trabajo subraya la necesidad de avanzar en el conocimiento respecto del desarrollo y especialización de cada uno de tales organismos en la región.

Centrado en un período postdictatorial, el artículo de Eva Muzzopappa y Celeste Schnyder apunta a interrogar dos fondos documentales que se encuentran actualmente en poder de la justicia federal. Producidos por los respectivos departamentos de inteligencia de la Policía de Santiago del Estero y de una base aeronaval ubicada en la provincia de Chubut hasta los años 2004 y 2006 respectivamente, se presentan como una oportunidad para indagar sobre las actividades de inteligencia en el marco del sistema democrático luego de la última dictadura en Argentina. El trabajo retoma entonces las categorías a partir de las cuales se constituyen y organizan estos archivos, así como las empleadas en los informes de inteligencia, para retomar algunas preguntas respecto del rol de los organismos de información e inteligencia en los sistemas democráticos y dar cuenta de los puntos de tensión entre las prácticas de inteligencia, los "usos y costumbres" y la legislación y normativa vigente.

Este dossier dialoga además con la entrevista publicada en la siguiente sección de la revista donde se conjuga lo que para muchas/os en el equipo ha sido un punto de partida en la reflexión teórico-metodológica sobre los archivos y las burocracias estatales. Convocamos para ello a la Dra. Sofía Tiscornia y la Dra. Lila Caimari, dos referentes de la antropología y la historia, a conversar respecto de cómo han trabajado sobre archivos policiales en sus respectivas trayectorias partiendo del reconocimiento de que sus proposiciones han producido una serie de líneas de investigación que conjugan aquello que aquí queremos destacar: un abordaje que contemple la mirada de producción documental en línea con los procesos de configuración de las burocracias de las instituciones y organismos que tienen, como función, la producción de inteligencia.

En ese sentido, esperamos que este dossier pueda aportar al crecimiento de ese campo de investigación capaz de incidir en la comprensión del pasado y el presente desde una mirada crítica, para la reconfiguración de expectativas de futuro, que la Facultad de Humanidades y Ciencias de la Educación de la Universidad Nacional de La Plata y la Comisión por la Memoria de la Provincia de Buenos Aires proyectaron al constituir la Maestría en Historia y Memoria de la que esta revista es parte.

\section{REFERENCIAS}

Bertoia, L. (21 de abril de 2021). Espionaje ilegal: El gobierno de Mauricio Macri espió a 354 personas y a 171 organizaciones políticas. Página 12. Recuperado de: https://www.pagina12.com.ar/336830-espionaje-ilegal-el-gobierno-de-mauricio-macri-espio-a-354-p?fbcli d=IwAR3rab5_rlz7yVE2OAkHgnGDtjpDEem3KEDp1r1hFu1RPbS5dxbTveE3-48

Fossa, L. (01 de noviembre de 2019). PacoLeaks: Estos son los nombres y organizaciones que han sido vigiladas por Carabineros en los últimos meses. Interferencia. Recuperado de: https://interferencia.cl/articulos/pacoleaks-est os-son-los-nombres-y-organizaciones-que-han-sido-vigiladas-por-carabineros-en 
González Quintana, A. (2009). Politicas archivisticas para la defensa de los derechos humanos. Actualizacióny ampliación del informe elaborado para la UNESCO y el Consejo Internacional de Archivos (1995) sobre gestión de los archivos de los servicios de seguridad del estado de los desaparecidos regimenes represivos. Santiago de Compostela: Fundación 10 de Marzo. Recuperado de: https://www.ica.org/es/politicas-archivisticas-para-la-defensa-de-los-derechos-h umanos

L'Heuillet, H. (2010). Baja politica, alta policía. Buenos Aires: Prometeo.

Muzzopappa, E. (2018). Secreto en el estado. Millitares, justicia e inteligencia en Trelew. Buenos Aires: Teseo. Recuperado de: https://www.teseopress.com/secretoenelestado/

Oszlak, O. y O'Donnell, G. (1976). Estado y politicas estatales en América Latina: hacia una estrategia de investigación. Documento G.E. CLACSO No 4. Buenos Aires: CEDES.

Sepúlveda, N. (29 de octubre de 2019). Hackeo a Carabineros en medio de la crisis expone 10.515 archivos: entre ellos hay datos de inteligencia. CIPER. Recuperado de: https://www.ciperchile.cl/2019/10/29/hackeo-a-carabinero s-en-medio-de-la-crisis-expone-10-515-archivos-entre-ellos-hay-datos-de-inteligencia/

Stoler, A. L. (2010). Archivos coloniales y el arte de gobernar. Revista Colombiana de Antropología, 46(2), 465-496.

\section{Notas}

1 Los documentos de la Delegación de Orden Político y Social de Paraná (1920 - 1989) (DEOPS) en el Archivo Público Estatal de Paraná, Curitiba; los Delegación de Orden Político y social de Ceará (1963 1987), en el Archivo Público del Estado de Ceará, Fortaleza; la Secretaría de Seguridad Pública (1954 1989) (DOPS) en el Archivo Histórico de Río Grande do Sul, Porto Alegre; la Delegación de Orden Político y Social $(1926$ 1991) en el Archivo Público Estatal de Jordão Emerenciano, Recife; el Departamento Autónomo de Orden (1934 1975) y el Político y Social del Estado de Río de Janeiro y el Departamento de Orden Político y Social del Estado de Guanabara (1935 1982) en el Archivo Público del Estado de Río de Janeiro; de la Delegación de Orden Político y Social de Maranhão (1968 1991) en el Archivo Público del Estado de Maranhão de São Luís y de la Delegación de Orden Político y Social de Espíritu Santos (DOPS/ES) (1964 1985) en el Archivo Público del Estado de Espíritu Santo, Vitória.

2 Para ampliar esta información puede consultarse González Quintana (2009) y la Guía sobre archivos y fondos documentales vinculados con las graves violaciones a los derechos humanos cometidas en el marco de las coordinaciones represivas del Cono Sur en el pasado reciente publicada por el Instituto de Políticas Públicas en Derechos Humanos del MERCOSUR (IPPDH): http://atom.ippdh.mercosur.int/index.php/ 